\title{
Síndrome de Budd-Chiari de aparición en el puerperio: reporte de caso y revisión de la literatura
}

\section{Case report and literature review of Budd-Chiari syndrome during the puerperium}

Gabriel Sebastián Díaz, ${ }^{1}$ Carlos Alberto Salgado, ${ }^{2}$ Adolfo Zuluaga, ${ }^{3}$ Estefanía Orozco, ${ }^{3}$ Juan Ignacio Marín. ${ }^{4 *}$

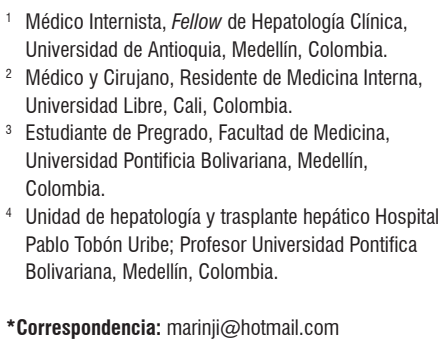

${ }^{4}$ Unidad de hepatología y trasplante hepático Hospita Pablo Tobón Uribe; Profesor Universidad Pontifica Bolivariana, Medellín, Colombia.

*Correspondencia: marinji@hotmail.com

\begin{abstract}
Resumen
El síndrome de Budd-Chiari es una entidad definida como la obstrucción al flujo sanguíneo en el tracto de salida hepático. Esta obstrucción se puede localizar desde las pequeñas venas hepáticas hasta la entrada de la vena cava inferior a la aurícula derecha. En la mayoría de los casos, se ocasiona por una trombosis endoluminal conocida como Budd-Chiari primario y secundario, cuando ocurre por una compresión extrínseca asociada a las lesiones que ocupan espacio como malignidad. Por tanto, los estados trombofilicos hereditarios se conocen como los principales factores de riesgo. El embarazo y el puerperio, al ser estados hipercoagulables, se pueden asociar al síndrome de Budd-Chiari, sin embargo, la prevalencia reportada en la literatura es muy variable dependiendo de la población estudiada. En Colombia no hay estudios de incidencia o prevalencia de la enfermedad; existen varios reportes de caso, pero no relacionados con el embarazo. Reportamos el caso de una paciente, que 12 semanas posparto presentó un síndrome de Budd-Chiari; además presentamos el manejo ofrecido, la evolución clínica y una revisión de la literatura de los casos asociados con el embarazo.
\end{abstract}

\section{Palabras clave}

Síndrome de Budd-Chiari, periodo posparto, trombosis de la vena.

\section{Abstract}

Budd-Chiari syndrome is defined as obstruction of hepatic blood outflow. This obstruction can be located anywhere from the small hepatic veins at the entrance of the inferior vena cava to the right atrium. Most cases are primary Budd-Chiari syndrome which is caused by endoluminal thrombosis. Secondary Budd-Chiari syndrome occurs as the result of extrinsic compression associated with space-occupying lesions such as malignant tumors. Hereditary thrombophilic states are the main risk factors, but since pregnancy and the puerperium are hypercoagulable states, they can be associated with Budd-Chiari syndrome. Nevertheless, the prevalence of this type of case in the literature varies according to the population studied. There have been no studies on the incidence or prevalence of this disease in Colombia. The small number of case reports here have not been related to pregnancy.

We report the case of a patient who developed Budd-Chiari syndrome 12 weeks postpartum. Our report includes management and clinical evolution as well as a review of the literature of cases associated with pregnancy.

\section{Keywords}

Budd-Chiari syndrome, postpartum period, venous thrombosis. 


\section{INTRODUCCIÓN}

El síndrome de Budd-Chiari se define como la condición en la que el flujo venoso hepático se encuentra obstruido a cualquier nivel, desde las venas hepáticas hasta el sitio de unión con la vena cava inferior y la aurícula derecha (1). Este, a su vez, puede dividirse en primario y secundario: primario, si la afectación es vascular intraluminal, generalmente por trombosis; o secundario, debido a una compresión extrínseca del lecho venoso descrito. Se acepta que la obstrucción secundaria a cirugía, como el trasplante o la resección hepáticos, la cirugía cardíaca, la compresión extrínseca o la invasión tumoral deben ser consideradas como causas secundarias (2). Su epidemiologia a nivel mundial es muy variable. Mientras que en países europeos como Dinamarca se evidencia una incidencia del 0,5 por cada millón de habitantes-año (3), en Japón, la prevalencia alcanza a ser del 2,4 por millón de habitantes, con aproximadamente 20 nuevos casos cada año (4). Sus factores de riesgo se encuentran establecidos, dentro de los cuales cabe resaltar los síndromes mieloproliferativos, el síndrome antifosfolípido, la hemoglobinuria paroxística nocturna, la hiperhomocisteinemia, la mutación del factor $\mathrm{V}$ Leiden y el gen de la protrombina (G20210A), las deficiencias de las proteínas $\mathrm{C}$ y S, el embarazo, el puerperio, la pobreza y la historia familiar (2). Particularmente, el embarazo es un estado fisiológico hipercoagulable en preparación para el parto, lo cual aumenta el riesgo de eventos tromboembólicos durante esta etapa y el posparto, con una incidencia que es 7-10 veces mayor cuando se compara con controles de la misma edad $(5,6)$. De igual manera, existe evidencia clínica de que el riesgo de eventos trombóticos va más allá del parto, en el puerperio, con una incidencia mucho mayor que los controles no embarazadas (7). La prevalencia reportada en la literatura del síndrome de Budd-Chiari relacionado con el embrazo y el puerperio es muy variable (8). En este artículo reportamos el caso de un síndrome de Budd-Chiari posparto, a través del cual resaltamos la importancia de tener en cuenta el embarazo y el puerperio como un factor de riesgo en este grupo de pacientes.

\section{DESCRIPCIÓN DEL CASO CLÍNICO}

Se trata de una paciente de 14 años, de raza negra, procedente del área urbana de Quibdó, Chocó, quien tiene historia de parto vértice espontáneo a término sin complicaciones y anticoncepción posparto con acetato de medroxiprogesterona de depósito, sin otros antecedentes médicos relevantes. En el segundo mes posparto inicia cuadro de dolor abdominal generalizado, aumento del perímetro abdominal e ictericia, sin embargo, no consultó hasta un mes después del inicio de los síntomas, cuando presentó un episodio de hematemesis. Al examen físico, como hallazgos relevantes, se evidenciaron ictericia en escleras, ascitis y hepatomegalia palpable a 4 centímetros del borde costal derecho. Se iniciaron estudios, y la endoscopia digestiva mostró una gastropatía hipertensiva y várices esofágicas

Tabla 1. Resultados de laboratorio

\begin{tabular}{lcc}
\hline \multicolumn{1}{c}{ Laboratorio } & Resultado & Valores de referencia \\
\hline ALT & 11 & $0-35 \mathrm{U} / \mathrm{L}$ \\
AST & 27 & $0-35 \mathrm{U} / \mathrm{L}$ \\
Bilirrubina total & 2,82 & $0,3-1 \mathrm{mg} / \mathrm{dL}$ \\
Bilirrubina directa & 1,92 & $0,1-0,3 \mathrm{mg} / \mathrm{dL}$ \\
Fosfatasa alcalina & 53 & $30-120 \mathrm{U} / \mathrm{L}$ \\
GGT & 72 & Hasta $40 \mathrm{U} / \mathrm{L}$ \\
Creatinina & 0,79 & $<1,5 \mathrm{mg} / \mathrm{dL}$ \\
Sodio & 140 & $136-145 \mathrm{mEq} / \mathrm{L}$ \\
Potasio & 3,47 & $3,5-5 \mathrm{mEg} / \mathrm{L}$ \\
Albúmina & 6,1 & $3,5-5,5 \mathrm{~g} / \mathrm{dL}$ \\
Leucocitos & 7700 & $4500-11000 / \mathrm{mm}^{3}$ \\
Hemoglobina & 9 & $12-16 \mathrm{~g} / \mathrm{dL}$ \\
Hematocrito & 27 & $36-46 \%$ \\
Neutrófilos & $50 \%$ & $40-70 \%$ \\
Linfocitos & $34 \%$ & $22-44 \%$ \\
Plaquetas & 238000 & $150000-350 \mathrm{000} / \mathrm{mm}^{3}$ \\
PT & 21,1 & $11,1-13,1 \mathrm{seg}$ \\
PTT & 42 & $22,1-35,1 \mathrm{seg}$ \\
INR & 1,99 & $0,9-1,2$ \\
AgsHB & Negativo & $\mathrm{NR}$ \\
Ac-VHC & $0,9 \mathrm{IU} / \mathrm{mL}$ & $0,8-1,2 \mathrm{IU} / \mathrm{mL}$ \\
ANA & &
\end{tabular}

Ac: anticuerpo; Ac-VHC: anticuerpos contra el virus de hepatitis C; AgsHB: antígeno de superficie virus de hepatitis B; ALT: alanina aminotransferasa; ANA: anticuerpos antinucleares; AST: aspartato aminotransferasa; DRVVT: Prueba de Russell; GGT: gamma glutamil transpeptidasa; IgA: inmunoglobulina A; IgG: inmunoglobulina G; IgM: inmunoglobulina M; INR: International Normalized Ratio; NR: no reactivo; PT: tiempo de protombina; РTT: tiempo de tromboplastina parcial. 
grado II, las cuales se ligaron endoscópicamente. Requirió paracentesis evacuante en dos oportunidades (5000 y 7000 $\mathrm{mL}$, respectivamente), y el líquido ascítico con gradiente de albúmina elevado. El estudio Doppler de circulación hepática mostró ausencia de flujo a nivel de las venas suprahepáticas y la tomografía de abdomen mostró cambios de hipertensión portal (circulación colateral y ascitis), con trombosis extensa de las venas suprahepáticas y hepatomegalia con efecto compresivo a nivel de la vena cava (Figura 1), lo que confirmaba el diagnóstico de síndrome de BuddChiari subagudo posparto. Los estudios de laboratorio se describen en la Tabla 1. El puntaje de Rotterdam (índice de pronóstico en Budd-Chiari) fue de 1,16, lo que la ubicó en riesgo intermedio. Se ampliaron los estudios en busca de una trombofilia que explicara la etiología del cuadro clínico, pero ninguno de estos fueron positivos. Esto confirmó la relación causal del puerperio como estado hipercoagulable y el síndrome de Budd-Chiari, y se consideró probable que el uso de medroxiprogesterona potenciara la condición trombótica.

La paciente se llevó a hemodinamia hepática y cavografía, en donde se encontró una oclusión crónica total del tracto hepático de salida con derivación del flujo por una colateral y un gradiente de presión venosa hepática (GPVH) de 22 $\mathrm{mm} \mathrm{Hg}$ sin posibilidad de manejo con stent, por lo que se colocó una derivación portosistémica transyugular intrahepática (transjugular intrahepatic portosystemic shunt, TIPS) Viatorr de $10 \times 70 \mathrm{~mm}$, que requirió, además, una extensión proximal con stent autoexpansible de $10 \times 60 \mathrm{~mm}$. El control angiográfico reveló desaparición de las várices, y el GPVH
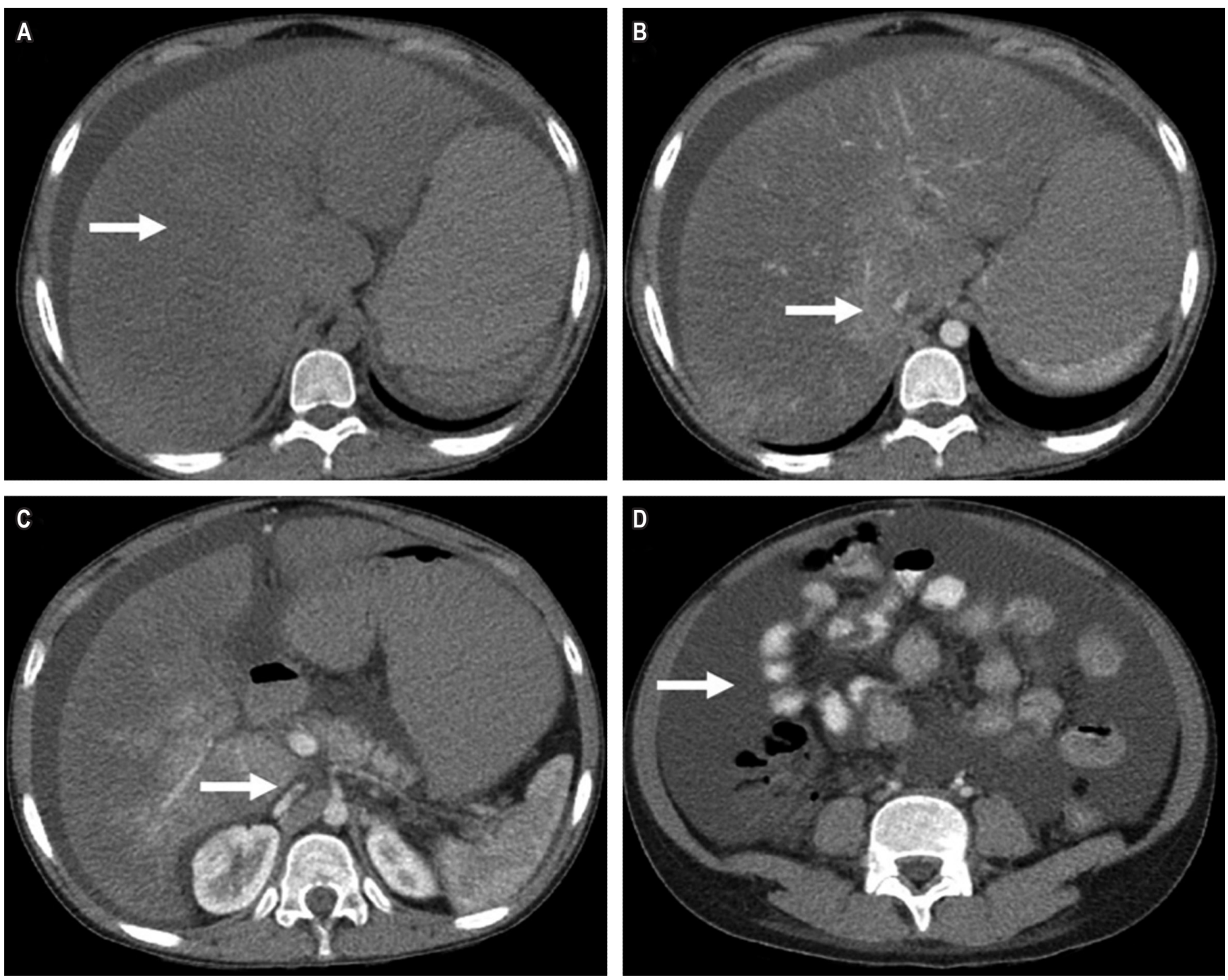

Figura 1. Tomografía de abdomen y pelvis. A. Fase sin contraste, hepatomegalia. B. Fase contrastada, ausencia de flujo en vena suprahepáticas. C. Fase contrastada, compresión de la vena cava inferior. D. Fase contrastada, ascitis. 
pos-TIPS fue de $8 \mathrm{~mm} \mathrm{Hg}$ (Figura 2). Después del procedimiento, la paciente inicia una mejoría clínica progresiva, con mejoría del dolor abdominal, control de la ascitis con terapia diurética y sin necesidad de una nueva paracentesis; además, presenta descenso de las aminotransferasas y de la bilirrubina. Es dada de alta e inicialmente se le prescribe anticoagulación con heparinas de bajo peso molecular. En dos controles ambulatorios posteriores que ha tenido la paciente se encontró clínicamente bien, sin ascitis, con resolución de la hepatomegalia, perfil hepático normal y permeabilidad del TIPS en 2 estudios Doppler de seguimiento.
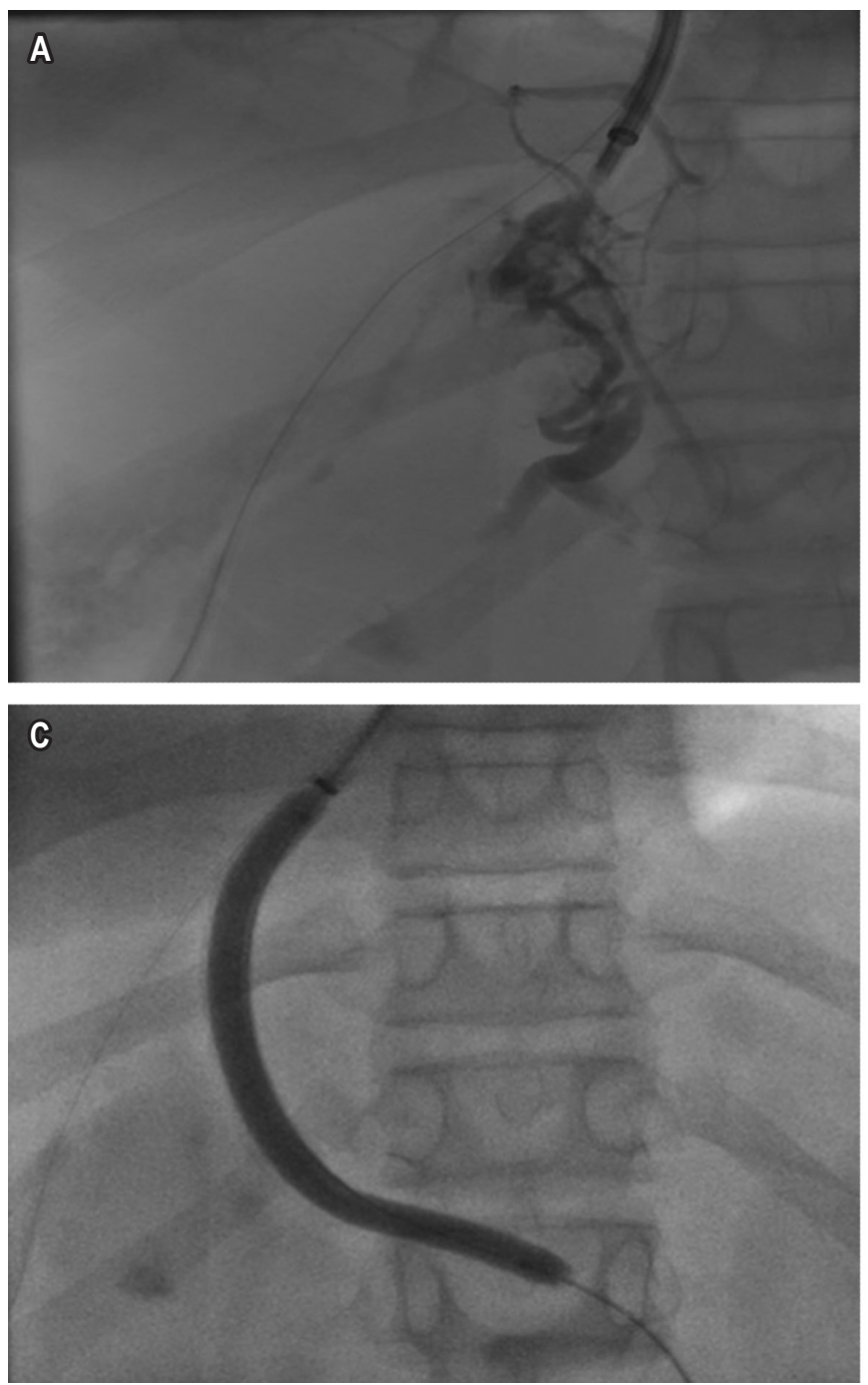

El período posparto se encuentra asociado con un incremento del riesgo de presentar eventos trombóticos (9). A pesar de que la definición de puerperio se encuentra actualmente en 6 semanas posterior al alumbramiento, se han presentado estudios que indican que existe un riesgo elevado de presentar manifestaciones asociadas a la trombosis durante el período de posparto, inclusive hasta las 12 semanas después del mismo (9). Diferentes estudios reportan complicaciones trombóticas como infarto de miocar-
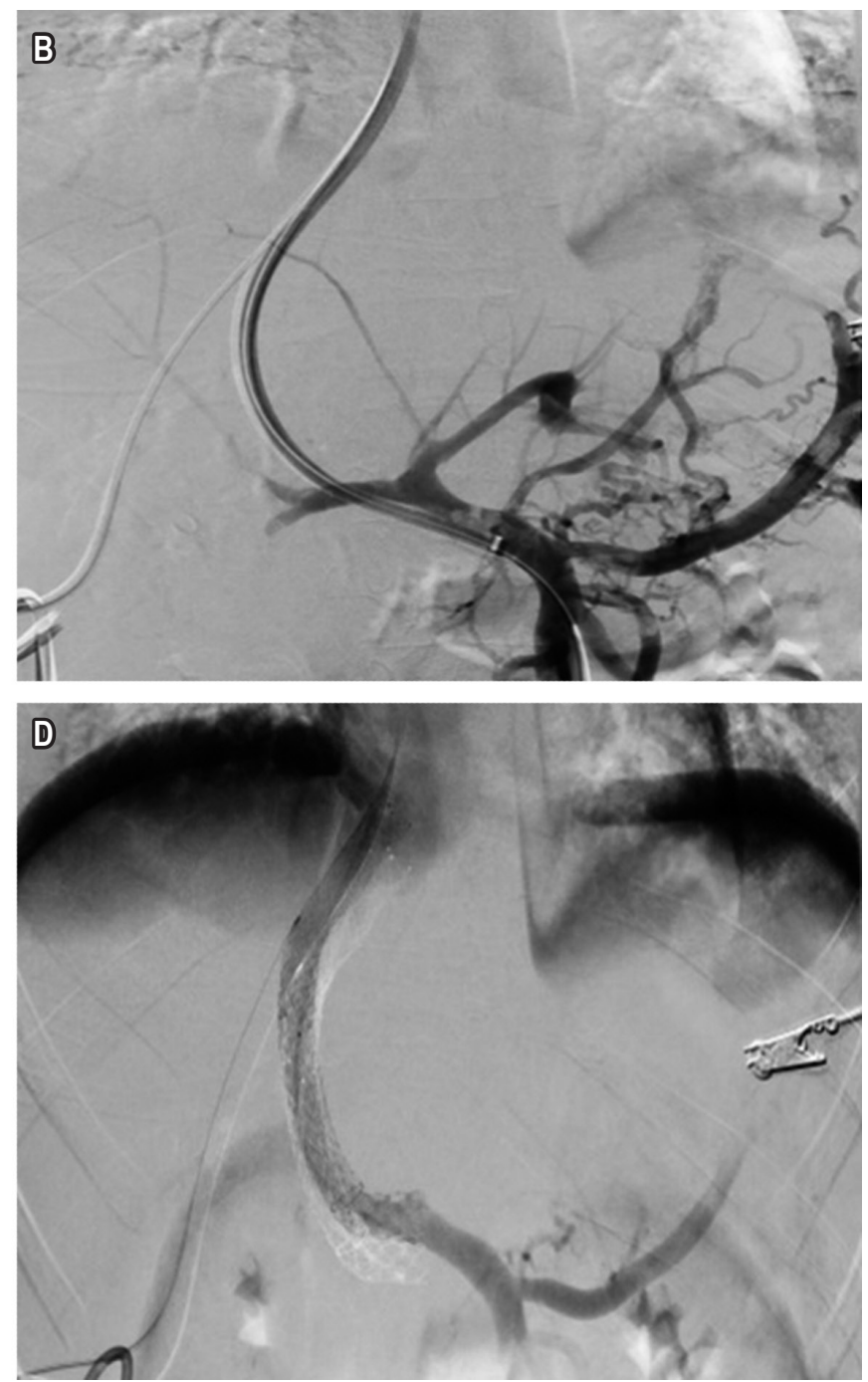

Figura 2. Implante de TIPS. A. Obstrucción completa de las venas suprahepáticas con derivación del flujo por colaterales. B. Permeabilidad de la vena porta y sus ramas, presencia de várices esofágicas. C. Dilatación con balón del trayecto de la TIPS. D. TIPS Viatorr de $10 \times 70 \mathrm{~mm}$ con extensión proximal con stent autoexpansible de $10 \times 60 \mathrm{~mm}$, desaparición de várices esofágicas. 
dio, accidente cerebrovascular y tromboembolismo venoso durante el puerperio (10-12); sin embargo, los reportes de síndrome de Budd-Chiari relacionados con el embarazo y el puerperio son muy variables (8). Una revisión sistemática reciente que reunió 120 pacientes con síndrome de Budd-Chiari relacionados con el embarazo encontró una prevalencia de este síndrome de 6,8 \% (6). Esto ubica al embarazo como estado hipercoagulable, con una prevalencia similar a otros factores de riesgo conocidos como la mutación del factor $\mathrm{V}$ Leiden, el gen de la protrombina G20210A y otras trombofilias. Por lo tanto, cuando se está evaluando la etiología en un caso de Budd-Chiari, se debe considerar al embarazo como un factor de riesgo.

La revisión sistemática descrita reúne pacientes de 20 países, predominantemente de Asia y Europa (6). Aunque el reporte de este caso es importante, la información acerca de la prevalencia real de los factores de riesgo para BuddChiari en Colombia requiere un estudio colaborativo de los centros de referencia donde evaluamos a los pacientes con patologías vasculares hepáticas. Existen, incluso, diferencias culturales en cuanto al manejo del puerperio. En la India, por ejemplo, donde hay reportes de prevalencia de Budd-Chiari relacionado con el embarazo tan altos como $13,1 \%$, esto parece no solo estar relacionado con el estado hipercoagulable puerperal, sino también con períodos de reposo posparto entre 30 y 40 días, con acceso limitado a una buena hidratación (13). Una creencia similar existe todavía en Colombia, sobre todo en áreas rurales.

La forma clínica de presentación más común de BuddChiari asociado con el embarazo es aguda y por trombosis de las venas suprahepáticas con obstrucción al tracto de salida (14). Esta fue la presentación del caso que estamos reportando, aunque advertimos que, por dificultades sociales, la paciente consultó de manera tardía.

La mejor comprensión de la enfermedad y el desarrollo de nuevos tratamientos ha permitido modificar la historia natural del paciente con síndrome de Budd-Chiari. El algoritmo de tratamiento paso a paso validado en diferentes cohortes $(15,16)$ propone, de acuerdo con la situación de cada paciente en particular y los hallazgos en la hemodinamia hepática, ir desde el manejo inicial con anticoagulación, la angioplastia o un stent con trombólisis a la colocación de una TIPS o a la realización de un trasplante de hígado en el caso de una falla hepática aguda, insuficiencia hepática crónica o cuando el puntaje de Rotterdam es adverso. En el caso que reportamos, los hallazgos en hemodinamia de una trombosis crónica, con oclusión completa del tracto de salida, indicaron la colocación de una TIPS; y gracias a la disponibilidad de este dispositivo, se logró la descomprensión del tracto de salida hepático y la recuperación clínica descrita en la presentación del caso clínico.
Finalmente, con este caso queremos resaltar que se debe considerar al embarazo y al puerperio como estados hipercoagulables y factores de riesgo para eventos vasculares hepáticos en este grupo de población específico.

\section{REFERENCIAS}

1. DeLeve LD, Valla DC, Garcia-Tsao G; American Association for the Study Liver Diseases. Vascular disorders of the liver. Hepatology. 2009 May;49(5):1729-64. doi: 10.1002/ hep. 22772.

2. Qi X, Han G, Guo X, De Stefano V, Xu K, Lu Z, et al. Review article: the aetiology of primary Budd-Chiari syndrome - differences between the West and China. Aliment Pharmacol Ther. 2016 Dec;44(11-12):1152-1167. doi: 10.1111/apt.13815.

3. Almdal TP, Sørensen TI. Incidence of parenchymal liver diseases in Denmark, 1981 to 1985: analysis of hospitalization registry data. The Danish Association for the Study of the Liver. Hepatology. 1991 Apr;13(4):650-5. doi: 10.1016/0270-9139(91)92559-Q.

4. Okuda H, Yamagata H, Obata H, Iwata H, Sasaki R, Imai F, et al. Epidemiological and clinical features of Budd-Chiari syndrome in Japan. J Hepatol. 1995 Jan;22(1):1-9. doi: 10.1016/0168-8278(95)80252-5.

5. Falter HJ. Deep vein thrombosis in pregnancy and the puerperium: a comprehensive review. J Vasc Nurs. 1997 Jun;15(2):58-62. doi: 10.1016/S1062-0303(97)90002-9.

6. Ray JG, Chan WS. Deep vein thrombosis during pregnancy and the puerperium: a meta-analysis of the period of risk and the leg of presentation. Obstet Gynecol Surv. 1999 Apr;54(4):265-71.

7. Mahmoodi BK, Brouwer JL, Ten Kate MK, Lijfering WM, Veeger NJ, Mulder AB, et al. A prospective cohort study on the absolute risks of venous thromboembolism and predictive value of screening asymptomatic relatives of patients with hereditary deficiencies of protein S, protein $\mathrm{C}$ or antithrombin. J Thromb Haemost. 2010 Jun;8(6):1193200. doi: 10.1111/j.1538-7836.2010.03840.x.

8. Ren W, Li X, Jia J, Xia Y, Hu F, Xu Z. Prevalence of BuddChiari Syndrome during Pregnancy or Puerperium: A Systematic Review and Meta-Analysis. Gastroenterol Res Pract. 2015;2015:839875. doi: 10.1155/2015/839875.

9. Kamel H, Navi BB, Sriram N, Hovsepian DA, Devereux RB, Elkind MS. Risk of a thrombotic event after the 6-week postpartum period. N Engl J Med. 2014 Apr 3;370(14):130715. doi: 10.1056/NEJMoa1311485.

10. Sultan AA, West J, Tata LJ, Fleming KM, Nelson-Piercy C, Grainge MJ. Risk of first venous thromboembolism in and around pregnancy: a population-based cohort study. $\mathrm{Br} \mathrm{J}$ Haematol. 2012 Feb;156(3):366-73. doi: 10.1111/j.13652141.2011.08956.x.

11. James AH, Bushnell CD, Jamison MG, Myers ER. Incidence and risk factors for stroke in pregnancy and the puerpe- 
rium. Obstet Gynecol. 2005 Sep;106(3):509-16. doi: 10.1097/01.AOG.0000172428.78411.b0.

12. Heit JA, Kobbervig CE, James AH, Petterson TM, Bailey KR, Melton LJ 3rd. Trends in the incidence of venous thromboembolism during pregnancy or postpartum: a 30-year population-based study. Ann Intern Med. $2005 \mathrm{Nov}$ 15;143(10):697-706. doi: 10.7326/0003-4819-143-10200511150-00006.

13. Dilawari JB, Bambery P, Chawla Y, Kaur U, Bhusnurmath SR, Malhotra HS, et al. Hepatic outflow obstruction (Budd-Chiari syndrome). Experience with 177 patients and a review of the literature. Medicine (Baltimore). 1994 Jan;73(1):21-36. doi: 10.1097/00005792-19940100000003.
14. Rautou PE, Plessier A, Bernuau J, Denninger MH, Moucari R, Valla D. Pregnancy: a risk factor for Budd-Chiari syndrome? Gut. 2009 Apr;58(4):606-8. doi: 10.1136/ gut.2008.167577.

15. Plessier A, Sibert A, Consigny Y, Hakime A, Zappa M, Denninger $\mathrm{MH}$, et al. Aiming at minimal invasiveness as a therapeutic strategy for Budd-Chiari syndrome. Hepatology. 2006 Nov;44(5):1308-16. doi: 10.1002/hep.21354.

16. Seijo S, Plessier A, Hoekstra J, Dell'era A, Mandair D, Rifai $\mathrm{K}$, et al. Good long-term outcome of Budd-Chiari syndrome with a step-wise management. Hepatology. 2013 May;57(5):1962-8. doi: 10.1002/hep.26306. 